\title{
MedienPädagogik
}

www. medienpaed.com

\section{Das Fernsehen als Kummertante - Beratungsangebote für Kinder}

\author{
Sandra Fleischer
}

\begin{abstract}
Mehrere Sender buhlen mit Beratungsversprechen und ominösen Lebenshilfetipps um Zuschauer. Die Super-Nanny, Hunde-Mutti, Hausfrau-Fee - alle wissen, wie es besser geht und «beraten» gern. Abseits von Pseudo-Beratungsshows, Tarotkarten und Aromastäbchentherapie bietet der KI.KA seit 2003 ein explizites Beratungsangebot für Kinder an. Das Projekt führt der KI.KA in Zusammenarbeit mit der Nummer gegen Kummer und der BZgA durch. Der KI.KA-Kummerkasten ist das erste interaktiv angelegte Beratungsangebot für Kinder ab 7 Jahren im deutschen Fernsehen, allerdings nicht das erste mediale Beratungsangebot für Kinder in der Geschichte des Fernsehens. Der folgende Artikel bezieht sich auf Forschungsergebnisse, welche ich innerhalb meines Promotionsprojektes ${ }^{1}$ erarbeitete.
\end{abstract}

\section{Kinder auf Orientierungssuche in Fernsehinhalten}

Kinder sind intelligent und selbstbestimmt, sie suchen mit den Motiven der Neugier, des Wissensdursts und der Bewältigung von Problemstellungen nach Anregungen in ihrer Umwelt. Zu dieser Umwelt gehören die Medien, und im Medienalltag ist es für Kinder an erster Stelle das Fernsehen, dass auf dieser Suche von den Kindern funktionalisiert wird. ${ }^{2}$

Ausgehend von dieser Tatsache ist zu fragen, wie Kinder Beratungsangebote im Fernsehen wahrnehmen und verstehen, in welchen Lebenssituationen sich Kinder den Beratungsangeboten ${ }^{3}$ zuwenden sowie welche Bedeutung sie den dort gegebenen Tipps und Ratschlägen für ihr Leben geben.

Sollen diese Fragen beantwortet werden, so muss grundlegend auch geklärt werden, wie Beratung über Medien realisiert wird und ob die bewährten Beratungs-

Fleischer, Sandra (2006): «Mediale Beratungsangebote als Orientierungsquellen für Kinder. Ein Beitrag zur Theorie der Orientierungsfunktion des Fernsehens» Universität Leipzig, Dissertation.

2 Vgl. u. a. Baacke, Dieter (1993): Die 6- bis 12-Jährigen: Einführung in Probleme des Kindesalters. 5. Auflage. Weinheim und Basel.; Baacke, Dieter; Frank, Günter; Radde, Martin (1991): Medienwelten - Medienorte. Jugend und Medien in Nordrhein-Westfalen. Opladen.; Medienpädagogischer Forschungsverbund Südwest (Hrsg.) (2005): KIM-Studie 2005. Kinder und Medien. Computer und Internet. Basisuntersuchung zum Medienumgang 6- bis 13-Jähriger in Deutschland. Baden-Baden.

3 Mediale Beratungsangebote sind anhand von vier Merkmalen bestimmt: es sind Angebote, a) die von Macherseite aus auf der Ebene der Gesamtkonzeption das Ziel der Hilfestellung und Unterstützung für die Rezipienten erkennen lassen, b) die eine zentrale Person als Berater/Experte präsentieren, c) deren Ausstrahlung regelmässig erfolgt, also nicht nur in speziellen Folgen, sondern als feste Rubrik innerhalb einer Sendung oder als eigenständiges, regelmässiges Format und d) die einen nicht-fiktionalen Gesamtcharakter haben (vgl. Fleischer 2006). 
formen- und Modelle aus Psychologie und Pädagogik für eine Beratung über Medien - und ganz speziell mit dem Augenmerk auf die Zielgruppe Kind - angemessen sind.

Als exemplarisches Beispiel wurde für das Projekt das interaktiv angelegte Beratungsangebot KI.KA-Kummerkasten (KI.KA) und dessen Nutzung, Wahrnehmung, Bewertung und Verarbeitung durch Kinder (anhand von zwölf Einzelfällen) untersucht. Dazu wurden eine qualitative Programmanalyse und leitfadengestützte Befragungen von Kindern, die den KI.KA-Kummerkasten kennen, und deren Müttern ausgewertet ${ }^{4}$.

\section{Beratung über das Fernsehen?}

Beratung ist im Alltag wie auf Ebene der professionellen Beratung eine zwischenmenschliche Beziehung, eine vertrauensvolle Kommunikation von Mensch zu Mensch. Eine Beratung ist ein Gespräch, das durch ein gegenseitiges Zuhören und das miteinander Sprechen und Aufeinanderbezugnehmen charakterisiert ist. Kritisch hinterfragt werden muss also, ob es Beratung, verstanden als kommunikativer Hilfeprozess, vermittelt über ein Massenmedium überhaupt geben kann. Als Ergebnis des Forschungsprojektes kann auf Basis der theoretischen Auseinandersetzung sowie der empirischen Forschungsergebnisse ausgesagt werden, dass Beratung über ein massenmediales Beratungsangebot mit interaktiver Charakteristik möglich ist. Im Falle des Projektes KI.KA-Kummerkasten kann eine Beratungssituation durch das Ansehen der Sendung entstehen sowie auch angeregt durch die Sendung via Telefon, Brief und E-Mail. Denn die in der Fernsehsendung präsentierten Gespräche zwischen Moderatoren und Zuschauern am Telefon bzw. die Gespräche zwischen Moderatoren und Studiogästen werden von den zuschauenden Kindern als direktes Kommunikationsangebot verstanden. Die Kinder können sich in die Gesprächspartner gedanklich hineinversetzen und so den Gesprächsinhalt auf sich beziehen und für sich interpretieren.

Für die befragten zwölf Kinder ist diese Form der Medienaneignung nachvollziehbar. Stellen die Kinder zum Sendungsthema einen persönlichen Bezug her, schlüpfen sie in die präsentierten Rollen und können so - obwohl vor dem Fernseher sitzend - Teil eines Beratungsgespräches sein. Sie haben dabei auch am Bildschirm sitzend das Gefühl «es persönlich ein bisschen gesagt zu bekommen» (Yasmin 11 Jahre). Da das Beratungsangebot KI.KA-Kummerkasten zudem noch Live-Telefonate anbietet ${ }^{5}$, E-Mails und Faxe verliest und auf diese antwortet, erscheint die

\footnotetext{
4 Das Promotionsprojekt griff zur vertieften Analyse dabei auf bereits verschriftete Befragungen zurück, die im Auftrag des KI.KA innerhalb einer Studie zur Akzeptanz des KI.KA-Kummerkasten von der Autorin geführt worden waren. Die besagte Studie wurde am Lehrstuhl für Medienpädagogik und Weiterbildung der Universität Leipzig unter Leitung von Prof. Dr. Schorb 2004 durchgeführt, der Bericht ist unveröffentlicht.

5 wie auch täglich Beratungsgespräche ausserhalb der Sendezeit am Telefon und seit 2005 am Sendetag auch per Chat mit Mitarbeiterlnnen der Nummer gegen Kummer
} 
Fernsehsendung den Kindern als tatsächliches Gesprächsangebot. Die Ergebnisse zeigen: Die befragten Kinder fühlen sich vom KI.KA-Kummerkasten angesprochen und haben das Gefühl, mit ihm auch direkt kommunizieren zu können und Antworten und Rat von ihm zu erhalten.

Das festgestellte Gefühl der Kinder, vom KI.KA-Kummerkasten wahrgenommen und gehört zu werden, basiert noch auf weiteren Aspekten der Sendungsgestaltung. Die Fernsehsendung richtet sich explizit an Kinder und bezieht diese konsequent in die Sendung ein. Es werden Themen behandelt, die die Kinder betreffen und von den Zuschauern als ihre Themen wiedererkannt werden. Das zuschauende Publikum wird von den Moderatoren in jeder Sendung mehrfach direkt als «ihr» oder «du» angesprochen, dazu blicken die Moderatoren die Kinder scheinbar auch direkt an (direkter Blick in die Kamera). Zudem kommen Kinder in der Sendung tatsächlich zu Wort per Telefon, über verlesenen E-Mails und Briefe oder als Studiogäste. In jeder Sendeminute wird den Kindern Zuhause so vermittelt: Hier geht es um euch.

Dass sich Kinder tatsächlich angesprochen fühlen und die Sendung als Gesprächsangebot verstehen, zeigen nicht nur die Aussagen der befragten Kinder, sondern auch die enorme Anzahl von eingehenden Anrufen und schriftlichen Kommunikaten von Kindern bei der Redaktion des KI.KA. Bereits 2003, als der KI.KA-Kummerkasten noch als Rubrik im Magazin KI.KA LIVE lief, schätzte KI.KA-Programmchef Frank Beckmann die Anzahl der eingehenden Meldungen zu KI.KA LIVE folgendermassen ein: «Tausende von Anrufen jeden Tag, täglich 1000 Faxe und Briefe und 2000 E-Mails und jeden Monat mehr als zwölf Millionen Zugriffe auf die Homepage [...]» (Beckmann 2003). ${ }^{6}$

\section{Vertrauen zu einer Fernsehsendung}

Mit Blick auf die zwölf qualitativ untersuchten Einzelfälle als Gruppe kann festgestellt werden, dass die befragten Kinder nach Hilfestellungen für die Bearbeitung von Entwicklungsaufgaben der Mittleren Kindheit und der Adoleszenz, zu Kritischen Lebensereignissen und gesellschaftlichen und aktuell persönlichen Problematiken im medialen Beratungsangebot suchen. Was tun bei Streit mit Freunden, fragen die jüngsten Kinder, auch erfahrene physische und psychische Gewalt ist ein Thema, auch Zukunftsängste und der Wunsch, sich über gesellschaftliche Ungleichheit zu unterhalten, sowie Fragen zur Normgerechtheit des eigenen Körpers werden formuliert. Liebeskummer, Mobbing und Fragen zur beruflichen Chancen und zur Sexualität bewegen die älteren Kinder ab 12 Jahren.

Beckmann, Frank (2003): Einzigartig in der deutschen Fernsehlandschaft: Fundierte Lebenshilfe für Kinder: Der KI.KA-Kummerkasten. Pressemappe des KI.KA zum Sendestart des KI.KA-Kummerkasten. 
Anhand der untersuchten zwölf Einzelfälle zeigt sich: Mehrere der Kinder verstehen und schätzen den KI.KA-Kummerkasten als Ansprechpartner und Berater, dabei werden besonders von jüngeren Kindern zwischen 7 und 10 Jahren keine beratungsleistenden Personen wahrgenommen, sondern das Beratungsprojekt selbst, der KI.KA-Kummerkasten, wird als der Berater wahr- und angenommen und zu ihm wird eine, als parasozial zu bezeichnende, Beziehung aufgebaut. Die Kinder nehmen das Beratungsangebot nicht als journalistisches Produkt wahr, sondern als eine Anlaufstelle für ihre Probleme und bringen diesem verinnerlichten Bild eines Beraters Vertrauen entgegen. Sie schreiben «dem Kummerkasten», rufen «ihn» an und bitten «ihn» um Hilfe. Sie vertrauen darauf, dass der KI.KA-Kummerkasten für sie da ist, dass sie die Kommunikationssituation mit ihm bestimmen können, dass privat Kommuniziertes nicht «weitergeplappert» wird und dass in der Sendung die Themen von Kindern behandelt werden. Sie vertrauen weiterhin darauf, dass diese Themenbehandlung ernsthaft und ausführlich geführt wird und dass ihnen gute, glaubhafte Ratschläge gegeben werden. Dass die Sendung KI.KA-Kummerkasten Moderatoren hat und innerhalb und ausserhalb der Sendung professionelle Berater auftreten, blenden die Kinder zwischen 7 und 10 Jahren in ihrer Wahrnehmung und Verarbeitung beinahe vollständig aus. Moderatoren und Berater fungieren nur als Gesicht und Stimme des KI.KA-Kummerkasten, an die Namen und das Aussehen der in der Sendung auftretenden erwachsenen Personen kann sich fast keines der Kinder zwischen 7 und 10 Jahren erinnern. Die fernseherfahreneren älteren Kinder erinnern und kennen Moderatoren und Berater und beziehen ihre Bewertungen konkret auf deren Art und Weise des Sprechens und der Sinnhaftigkeit der Ratschläge. Für fünf der Kinder ist eine über den KI.KA-Kummerkasten erfahrene Beratung und beratungsähnliche Erfahrungen belegbar.

\section{Eine Vertrauter und zugleich eine moralische Instanz für die jüngeren Kinder, Unterhaltungsshow und medialer Highway zu anderen Kindern für die älteren Kinder}

Mit Blick auf das soziale Netzwerk der Kinder hat das Angebot KI.KA-Kummerkasten als Orientierungsquelle unterschiedlichen Stellenwert. Für einige Kinder kann eine starke Bedeutung interpretiert werden, wenn Kinder ihn als letzte Möglichkeit, Hilfe zur Lösung eines Problems zu finden, wahrnehmen, nachdem ihnen die Mitlieder ihres sozialen Netzwerkes nicht helfen konnten. In diesen Fällen (alle im Alter zwischen 7 und 10 Jahren) wird das Angebot deutlich personalisiert und mit positiven Emotionen bedacht. Der KI.KA-Kummerkasten wird als Vertrauter wahrgenommen, als Helfer und auch als moralische Instanz, die entscheiden kann, was richtig und was falsch ist, und die dem Kind sagen kann, wie es sich verhalten soll. Die befragten Kinder zwischen 7 und 10 Jahren nehmen das Beratungsangebot sehr ernst. Den allen medialen Beratungsangeboten innewohnenden dualistischen 
Charakter von Unterhalt und Beratung nehmen sie nicht wahr. Die Unterhaltungsabsicht der Fernsehmacher erschliesst sich den Kindern bis 10 Jahren nicht.

In zwei Fällen ist die Funktion der moralischen Instanz mit der Funktion des Sprachrohrs verknüpft. Falko (9 Jahre aus niedrigem Anregungsmilieu ${ }^{7}$ und aus einer Familie mit finanziellen Problemen) und Sascha (7 Jahre aus hohem Anregungsmilieu mit starkem ökonomischen Hintergrund), zwei Jungen aus unterschiedlichen Ecken Leipzigs, leiden unter Demütigungen und körperlicher Gewalt in der Schule. Beide Jungen haben keine gleichaltrigen Freunde und bei beiden sind die Eltern und Lehrer ratlos. Beide Jungen bitten den KI.KA-Kummerkasten um Rat und auch darum, im Fernsehen zu sagen, wie sie nicht mehr behandelt werden möchten. Der KI.KA-Kummerkasten wird als Berater und zugleich als anonymes, veröffentlichendes Sprachrohr verstanden, dass ihren Leiden und ihren Ansprüchen an ihre Peiniger endlich Ausdruck geben soll.

Der KI.KA-Kummerkasten soll für Sascha «mal sagen, dass man teilen soll und nicht immer einen auslassen»; «nicht schlagen, so dass man Kummer hat / Nicht schlagen oder prügeln. / Einem Klassenkameraden nicht das Essen wegnehmen.» - «Also, alles das, was ich gesagt habe, gibt es bei mir an der Schule. Also das ist mir selber alles schon passiert.», gibt der Siebenjährige zu. Schon häufig versuchte Sascha den KI.KA-Kummerkasten telefonisch zu erreichen, kam aber nie durch. Und er generalisiert diese Erfahrung bereits in der Hinsicht, dass ein Durchkommen zum KI.KA-Kummerkasten so gut wie unmöglich sei. Damit ist eine grosse Problematik angerissen: Das von den Kindern wahrgenommene senderseitig gegebene Versprechen, da zu sein und zu antworten, kann nicht eingehalten werden.

Falko ergeht es ganz ähnlich und er ist emotional sehr belastet. Seine Mutter gab ihm den Tipp, er solle zurückschlagen und sich nicht alles gefallen lassen. Das half Falko nicht weiter. Er hat Angst, wieder «auszurasten» und dann «wie wild um mich zu schlagen», wie schon einmal. Also hofft er sehr darauf, dass der KI.KA-Kummerkasten recht bald zu seinen Themen spricht. «Hoffentlich kommt bald eins meiner Themen beim Kummerkasten.»

Teilweise ist die Bedeutung des Beratungsangebotes als Orientierungsquelle auch themenabhängig und wird für Kinder nur dann relevant, wenn andere Bezugspersonen Teil des Problems sind und daher nicht zu Rate gezogen werden können. So im Fall von der schon sehr reifen Laura (12), die ihre Mutter sehr liebt, aber nicht weiss, wie sie ihr sagen soll, dass sie nicht mit ihr das erste Mal zum Frauenarzt gehen möchte. Zur Hilfestellung bei dieser Thematik war ihr der KI.KA-Kummerkasten eine Hilfe. Und es gibt Kinder, die den KI.KA-Kummerkasten als potentielle, jedoch nicht aktuell-relevante Orientierungsquelle wahrnehmen. Für diese

Das Anregungsmilieu beschreibt den formalen Bildungsgrad der Eltern. Hohes Anregungsmilieu: mindestens ein Elternteil hat Abitur oder höhere Abschlüsse; niedriges Anregungsmilieu: kein Elternteil hat Abitur, sondern Haupt- oder Realschulabschluss (bzw. keinen Schulabschluss) mit oder ohne abgeschlossene Lehre. 
Kinder ist der KI.KA-Kummerkasten bislang ein reines Unterhaltungsangebot und auch eine Kommunikationsplattform. Denn neben der Orientierungssuche können auch Unterhaltungs- und Kommunikationsmotive festgestellt werden. So wird der KI.KA-Kummerkasten in seiner Intention Beratungsangebot zu sein, zwar von allen 12 Kindern erkannt, jedoch wenden sich dem Angebot nicht alle Kinder - und auch nicht bei jeder Rezeption - mit Orientierungsbedürfnissen zu. Einige der älteren Kinder (11 bis 14 Jahre) funktionalisieren das interaktive Angebot auch zur Unterhaltung, Selbstdarstellung und als Kommunikationsmöglichkeit mit anderen Kindern. Die älteren Kinder amüsieren sich durchaus beim Sehen der Sendung. Sie mögen es von anderen Kindern zu hören und diesen über die interaktiven Möglichkeiten auch antworten zu können.

\section{Weder Konkurrenz noch Ersatz für Eltern und Freunde}

Mediale Beratungsangebote werden von den Kindern demnach nicht als Ersatz für ihre zentralen Ansprechpartner begriffen. Sie sind ein zusätzliches Angebot, das in bestimmten Situationen an Bedeutung gewinnen kann. Dabei wird das Angebot der KI.KA-Kummerkasten von den Kindern im medialen Kontext bislang als einzigartig wahrgenommen. Sie kennen keine weiteren Beratungsangebote für Kinder. Die älteren Kinder (12 bis 14 Jahre) grenzen die Sendung auch deutlich und begründet von «reisserischen» (Marie 14 Jahre) Talkshows ab.

Ein mediales Beratungsangebot, auch personalisiert angeeignet, verdrängt keine existierenden Bezugspersonen und Ansprechpartner. Für Kinder mit wenigen Ansprechpartnern kann das mediale Beratungsangebot jedoch von hohem Wert sein, indem es Fenster zu anderen Kindern ist, ein Gruppensetting anbietet und sich eventuell noch als direkter Gesprächspartner vorschlägt.

Dabei ist den Kindern die gebotene Anonymität am Bildschirm oder die weitestgehende Anonymität bei der Nutzung interaktiver Kommunikationsmöglichkeiten sehr wichtig. Gefühle von Peinlichkeit, Unsicherheit und Abnormalität können die Zuwendung hin zu sonst vertrauten sozialen Bezugspersonen verhindern. Das mediale Beratungsangebot, das aus der Ferne, in der Sicherheit, sich nicht entblössen zu müssen, funktionalisiert wird, ist für Kinder eine angenehme Art der Orientierungssuche zu (aus ihrer Sicht) prekären Themen. Das Angebot der Anonymität ist, wie die Einzelfälle zeigen, vielen, besonders aber den älteren Kindern wichtig. Das Angebot, den KI.KA-Kummerkasten anzurufen, nehmen einige der Kinder gern an, jedoch gibt es auch Kinder, die die Öffentlichkeit der Fernsehsendung verstehen (und von der Möglichkeit nach der Sendezeit privat anzurufen nichts wissen) und die deshalb das Angebot anzurufen nicht annehmen. Obwohl sie sehr gern auch persönlich mit dem als Berater wahrgenommenen KI.KA-Kummerkasten reden möchten, verhindern Gefühle der Peinlichkeit und die Angst, im Fernsehen über die Stimme erkannt zu werden, die Hinwendung per Anruf. Diese Kinder 
möchten auf ihrer Orientierungssuche anonym und zugleich privat sein und ziehen deshalb die klassische Fernsehrezeptionssitiuation - das Zusehen - vor.

\section{Nutzenpotenzial}

Aus psychologischer und pädagogischer Sicht zeigen die Ergebnisse, dass Kinder über die Aneignung eines medialen Beratungsangebotes Zugang zu Beratung finden und somit lernen können, dass das Hilfesuchen und das Annehmen von Hilfe ein sinnvolles Handeln ist. Über die Aneignung eines medialen Beratungsangebotes

- kann bei den zuschauenden Kindern ein Bewusstsein geweckt werden, dass es professionelle Beratungsangebote gibt,

- kann den Kindern vermittelt werden, dass es bei Beratungsangeboten erwachsene, vertrauenswürdige Ansprechpartner gibt,

- kann den Kindern bewusst gemacht werden, dass sie sich einem Beratungsangebot zuwenden dürfen,

- kann Kindern deutlich werden, dass das Suchen und Annehmen von Hilfe ein sinnvolles Handeln ist,

- können Kinder Bestätigung und/oder ermutigende Unterstützung erfahren, indem sie wahrnehmen, dass sie nicht die einzigen Kinder mit Fragen und Problemen sind und dass es Hilfe und Antworten für sie gibt,

- können Kinder Beratung und beratungsähnliche Erfahrungen zu einem persönlich wahrgenommenen Thema erleben (am Bildschirm vermittelt als zusätzlich auch über Individualkommunikation am Telefon oder per Brief, E-Mail),

- können Kinder Selbstbestätigung erfahren über das Mitteilen von Meinungen und das Geben von Ratschlägen live in der Sendung,

- können Kinder Unterhaltung geniessen.

\section{Ansprüche von Kindern an ein mediales Beratungsangebot - ein Katalog}

Mediale Beratungsangebote sind dann für Kinder ein mögliches hilfreiches Angebot, wenn sie über die journalistischen Überlegungen zur Kindgerechtheit hinaus die Ansprüche von Kindern an eine Beratung respektieren und - soweit möglich - umsetzen. Der von der Autorin auf Basis der theoretischen Auseinandersetzung und der empirischen Untersuchung formulierte Katalog von Ansprüchen von Kindern an mediale Beratungsangebote kann im Rahmen eines Artikels nicht ausführlich und begründet geleistet werden. An dieser Stelle kann lediglich eine pointierte Aufstellung der 18 Punkte gegeben werden:

1. Kindgerechtheit bezüglich der Fernsehfähigkeiten der Altersgruppe

2. Kindgerechtheit bezüglich der Beratbarkeit von Kindern und die Akzeptanz von Kindern als kompetente Akteure mit eigenen Rechten. Die Qualitätsebe- 
nen einer Beratung mit Kindern ${ }^{8}$ sind als anzustrebende Zielqualitäten auch bei massenmedialen Beratungsangeboten zu setzen.

Ergänzend zu den grundlegenden Ansprüchen von Kindgerechtheit in der medialen Präsentation und bezüglich des Beratungskonzeptes und -vollzugs werden folgende Anforderungen gestellt:

3. Der Anspruch einer professionellen Beratung, Ergebnis- und Wirkqualitäten zu bieten, sollte in der Wichtigkeit die Einschaltquote dominieren.

4. Ein Angebot, welches ein Beratungsangebot sein will und sich nicht auf ein Entertainment-Education-Angebot reduzieren lassen möchte, muss die individuellen Themen von Kindern respektieren und den Kindern direkten Einfluss auf die Sendungsinhalte gewähren.

5. Ein Beratungsangebot sollte inhaltlich Wiederholungen zulassen, d. h. Themen immer wieder erneut aufgreifen und weiter bearbeiten.

6. Ein Beratungsangebot sollte sich am Normalen ausrichten. Da Kinder in Beratungsangeboten nach Versicherungen und auch nach für sie tauglichen Handlungsvorschlägen suchen, sollte eine Beratungsangebot nicht am Aussergewöhnlichen ansetzen.

7. Die Themen sollten ausführlich und ernsthaft behandelt werden. Kinder lehnen eine reisserische Präsentation ab.

8. Die Leistung eines Beratungsangebotes sollte es sein, neben der Darstellung und Hinterfragung einer Problemstellung, auch Kompetenzen zur Weiterverarbeitung und zur Stärkung des Publikums zu geben.

9. Die Ausstrahlung eines medialen Beratungsangebotes sollte regelmässig und verlässlich geschehen. Den Kindern muss es möglich sein, sich dem Angebot auch nach einer längeren Unterbrechung in ihrer Rezeption wieder zuwenden zu können.

10. Im Falle von Kommunikationsangeboten über Individualmedien wie Brief und Telefon ist die Transparenz der Zugänglichkeit, der Erreichbarkeit und der Wahrscheinlichkeit der Rückmeldungen in jeder Sendung erneut herzustellen.

11. Werden Kinder als kompetente Akteure mit eigenen Rechten verstanden, dann sollte ihnen auch das Recht am eigenen Wort und das Recht auf Privatsphäre zugesprochen werden. Das öffentliche Verlesen von als privat eingesendeten E-Mails, Briefen und Faxen ist als unangemessen zu betrachten.

12. Jedem Kind ist in der öffentlichen wie auch in der privaten Kommunikationssituation Anonymität zuzusichern.

13. Aus pädagogischer und ethischer Sicht ist es nicht vertretbar, Kindern kostenpflichtige Angebote zur Kommunikation zu unterbreiten.

8 Köster-Goorkotte, Irmgard; Chow, Sergio (2004): Beratungsprozesse mit Kindern. In: Engel, Frank; Nestmann, Frank; Sickendiek, Ursel (Hrsg.): Das Handbuch der Beratung. Band 1 Disziplinen und Zugänge. Tübingen. S. 257-267. 
14. Gemäss einer professionellen Beratung muss ein mediales Beratungsangebot ein Verständnis von Beratung präsentieren, welches sich als kurzzeitiger Arbeitspakt versteht, mit dem Ziel der Auflösung der Beziehung nach der Beratung.

15. Mediale Beratungsangebote sollten dialogische Sendungselemente bieten, die einerseits zuschauenden Kindern Rollenangebote unterbreiten, die übernommen werden können, und andererseits anrufenden Kindern Gesprächspartner anbieten.

16. Ein mediales Beratungsangebot braucht medial präsentierte Personen, die diesen dialogischen Charakter tragen.

17. Um einen Beratungsprozess zu unterstützen, muss transparent dargestellt werden, wer den Kindern Beratung anbietet, d. h., wer in der Rolle des Beraters agiert.

18. Der Zielgruppe muss das Gefühl vermittelt werden, im Mittelpunkt des Interesses zu stehen, ernst genommen zu werden und Ratschläge von kompetenter Seite zu erhalten. Um dies zu gewährleisten, muss den Kindern zugehört werden. Eine Reduzierung von Kindern als Stichwortgeber oder Überleitung zum nächsten Sendungselement ist unangemessen und entspricht nicht dem Konzept von Beratung.

Deutlich wurde durch die Forschungsergebnisse, dass sich das Versprechen, Kinder persönlich zu beraten, für sie stets erreichbar und immer da zu sein, den Anbietern als grosse Herausforderung stellt. Die Arbeit zeigt, Kinder bis $10 \mathrm{Jah}-$ ren nehmen dieses Versprechen wortwörtlich und ernst und entkoppeln es von Sendezeiten. Wird ihnen etwas persönlich versprochen, fordern sie es ein. Auch sind sie noch nicht in der Lage, den dualen Charakter von Unterhaltung \& Beratung eines medialen Beratungsangebotes zu entschlüsseln. Vielmehr blenden sie den Unterhaltungsaspekt der Sendungen weitestgehend aus und sprechen dem medialen Berater Kompetenz und den Status einer moralischen Instanz zu. Aus ethischer Perspektive weiter zu diskutieren bleibt das Problem zwischen der massenmedialen Öffentlichkeit und dem in Beratungstheorie und -praxis formulierten Richtlinie, der Zusicherung der Privatheit der im Beratungsgespräch geäusserten Inhalte. Wie die Ergebnisse zeigen, wird durch die Anbieter medialer Beratungsangebote bislang unzureichend berücksichtigt, dass Anonymität und Privatheit keine Synonyme sind. Anonymität und Privatheit sind Eigenschaften unterschiedlicher Qualität.

\section{Fernsehen als geeignete Plattform für mediale Beratung von Kindern}

Fernsehen als mediale Plattform für Beratungsangebote für Kinder kann als geeignet argumentiert werden. Das Fernsehen ist das Lieblingsmedium von Kindern und ebenso wichtig, wie es banal erscheint, ist die Tatsache, dass Kinder Zugang 
zum Fernsehen haben. Fernsehen ist das ihnen vorrangig vertraute Medium. Radio und Zeitschriften werden erst für Kinder ab ca. 10, 11 Jahren in der Mediennutzung relevanter, das Internet noch später, erst ab ca. 12 Jahren. Den Analyse- und Verarbeitungsfähigkeiten von Kindern kommt zudem entgegen, dass ihnen das Fernsehen in seiner Darstellung und den journalistischen Formen vertraut ist und eine Aneignung von Beratungsangeboten auf diesen Schemata ansetzen kann. Durch die vorliegende Arbeit wird belegt: Kinder nehmen mediale Beratungsangebote an und nutzen sie zu ihrer Orientierungssuche. Sie schätzen ein mediales Beratungsangebot, wenn für sie erkennbar Kinder im Mittelpunkt des Angebotes stehen. Das Fernsehen für das Anbieten von Beratungsangeboten für Kinder zu nutzen, erscheint daher sinnvoll.

\section{Literatur}

Avery, Robert K. (1990). Talk Radio: The Private-Public Catharsis. In: Gumpert, Gary; Fish, Sandra L. (Eds.). Talking to Strangers: Mediated Therapeutic Communication. Norwood, New Jersey. S. 87-97.

Baacke, Dieter (1993). Die 6- bis 12-Jährigen: Einführung in Probleme des Kindesalters. 5. Auflage. Weinheim und Basel.

Baacke, Dieter; Frank, Günter; Radde, Martin (1991). Medienwelten - Medienorte. Jugend und Medien in Nordrhein-Westfalen. Opladen.

Bachmair, Ben (1993). TV-Kids. Ravensburg.

Bachmair, Sabine; Faber, Jan; Hennig, Claudius; Kolb, Rüdiger; Willig, Wolfgang (1989). Beraten will gelernt sein: Ein praktisches Lehrbuch für Anfänger und Fortgeschrittene. 4. überarbeitete Auflage. München.

Barnlund, Dean C. (1990). Therapeutic Communication. In: Gumpert, Gary; Fish, Sandra L. (Eds.). Talking to Strangers. Mediated Therapeutic Communication. Norwood, New Jersey. S. 10-28.

Beckmann, Frank (2003). Einzigartig in der deutschen Fernsehlandschaft: Fundierte Lebenshilfe für Kinder: Der KI.KA-Kummerkasten. Pressemappe des KI.KA zum Sendestart des KI.KA-Kummerkasten.

Beckmann, Frank (2004). Wir wollen zuhören. KI.KA LIVE ab August im KI.KA. Pressemappe des KI.KA zum Sendestart des Formates KI.KA LIVE.

Beckmann, Frank (2005). KI.KA- Kinderfernsehen mit Mehrwert. In: televizion, 18/2005/2. S.14-17.

Beisenherz, Gerhard (2005). Wie wohl fühlst Du Dich? Kindliche Persönlichkeit und Umwelt als Quelle von Wohlbefinden und Unwohlsein bei Grundschulkindern. In: Alt, Christian (Hrsg.). Kinderleben - Aufwachsen zwischen Familie, Freunden und Institutionen. Band 1: Aufwachsen in Familien. Wiesbaden. S. 157-186.

Bente, Gary; Fromm, Bettina (1997). Affektfernsehen. Motive, Angebotsweisen und Wirkungen. Opladen.

Bley, Stefanie (2004). Bravo oder Pfui? Zur Qualität von Jugendzeitschriften. Fachjournalist, Nr. 11/2004. S. 11-13.

BMFSFJ (2005). 12. Kinder- und Jugendbericht. Berlin.

Brake, Anna (2005). Wohlfühlen in der Familie. Wie Mütter und 8- bis 9-Jährige Kinder ihr Zusammenleben bewerten. In: Alt, Christian (Hrsg.). Kinderleben - Aufwachsen zwischen 
Familie, Freunden und Institutionen. Band 1: Aufwachsen in Familien. Wiesbaden. S. 45-62.

Cathcart, Robert; Gumpert, Gary (1990). Mediated Interpersonal Communication: Toward a New Typology. In: Gumpert, Gary; Fish, Sandra L. (Eds.). Talking to Strangers. Mediated Therapeutic Communication. Norwood, New Jersey. S. 39-53.

Dietrich, Georg (1991). Allgemeine Beratungspsychologie. Göttingen.

Dreher, Eva; Dreher, Michael (1985). Wahrnehmung und Bewältigung von Entwicklungsaufgaben im Jugendalter. In: Oerter, Rolf (Hrsg.). Lebensbewältigung im Jugendalter. Weinheim. S. 31-61.

Engel, Frank (2004). Beratung und Neue Medien. In: Engel, Frank; Nestmann, Frank; Sickendiek, Ursel (Hrsg.). Das Handbuch der Beratung. Band 1: Disziplinen und Zugänge. Tübingen. S. 497-509.

Engel, Frank; Nestmann, Frank; Sickendiek, Ursel (2004). Beratung - Ein Selbstverständnis in Bewegung. In: Engel, Frank; Nestmann, Frank; Sickendiek, Ursel (Hrsg.). Das Handbuch der Beratung. Band 1: Disziplinen und Zugänge. Tübingen. S. 33-44.

Fleischer, Sandra (2006). Mediale Beratungsangebote als Orientierungsquellen für Kinder. Ein Beitrag zur Theorie der Orientierungsfunktion des Fernsehens. Dissertation. Universität Leipzig.

Filipp, Sigrun-Heide (1995). Ein allgemeines Modell für die Analyse kritischer Lebensereignisse. In: Filipp, Sigrun-Heide (Hrsg.). Kritische Lebensereignisse. 3. Auflage. Weinheim. S. 3-52.

Fish, Sandra L. (1990). Therapeutic Uses of the Telephone: Crises Intervention vs. Traditional Therapie. In: Gumpert, Gary: Fish, Sandra L. (Eds.). Talking to Strangers. Mediated Therapeutic Communication. Norwood, New Jersey. S. 154-169.

Gerö, Sandra (2005). Kriterien der Qualitätssicherung für Online-Beratungsangebote. In: merz, 49 Jg, Nr. 5. S. 48-50.

Gleich, Uli (1995). Die Beziehung von Fernsehzuschauern zu Medienpersonen - eine explorative Untersuchung. In: Arbinger, R.; Jäger, R. S. (Hrsg.). Zukunftsperspektiven empirisch-pädagogischer Forschung. Landau. S. 363-381.

Gleich, Uli; Burst, Michael (1996). Parasoziale Beziehungen von Fernsehzuschauern mit Personen auf dem Bildschirm. In: Medienpsychologie, Nr. 3. S. 182-200.

Göttlich, Udo; Nieland, Jörg-Uwe (2001). Inszenierungs- und Rezeptionsweisen von Daily Soaps. In: Göttlich, Udo; Krotz, Friedrich; Paus-Haase, Ingrid (Hrsg.). Daily Soaps und Daily Talks im Alltag von Jugendlichen. Opladen. S. 23-135.

Gurt, Michael (2005). Lebenshilfe aus der Flimmerkiste!? Das Fernsehprogramm als Ratgeber und Orientierungsquelle. In: merz, 49. Jg. Nr. 5. S. 15-20.

Havighurst, Robert James (1956). Research on the Developmental-Task Concept. In: The School Review, 64. S. 215-223.

Havighurst, Robert James (1972). Developmental Tasks and Education. 3., überarbeitete Auflage. New York.

Hiddemann, Frank (1996). Talk als säkulare Beichte. Jürgen Domian mit Eins Live Talk Radio in WDR 3. In: medienpraktisch, Heft 4/96. S. 29-32.

Hoffmann, Andrea Claudia (1998). Öffentlichkeit als Therapie? Die Motivation von DaytimeTalk-Gästen. München.

Hoffmann, Dagmar (2005). Intimitäten im Netz. Jugendliche suchen Hilfe bei Internetportalen für sexuelle Aufklärung. In: merz, 49. Jg. Nr. 5. S. 38-43.

Honig, Michael-Sebastian (1999). Entwurf einer Theorie der Kindheit. Frankfurt am Main. 
Horton, Donald; Wohl, Richard R. (1956). Mass Communication and Para-social Interaction: Observation on Intimacy at a Distance. In: Psychiartry, 19. S. 215-229.

Janssen, Ludwig (1998). Auf der virtuellen Couch. Selbsthilfe, Beratung und Therapie im Internet. In: Janssen, Ludwig (Hrsg.). Auf der virtuellen Couch. Selbsthilfe, Beratung und Therapie im Internet. Bonn. S. 7-11.

Keupp, Heiner (2004). Beratung als Förderung von Identitätsarbeit in der Spätmoderne. In: Engel, Frank; Nestmann, Frank; Sickendiek, Ursel (Hrsg.). Das Handbuch der Beratung. Band 1: Disziplinen und Zugänge. Tübingen. S. 469-485.

Klein, Alexandra (2001). Medien der Sexualaufklärung einschliesslich des Internet. Eine qualitative Untersuchung mit Jugendlichen. Unveröffentlichte Diplomarbeit. Freie Universität Berlin.

Klein, Alexandra (2003). «Musst dich nicht schämen?». Sexuelle Aufklärung Jugendlicher und die Bedeutung medialer Angebote. In: tv diskurs, 23. S. 58-61.

Köster-Goorkotte, Irmgard; Chow, Sergio (2004). Beratungsprozesse mit Kindern. In: Engel, Frank; Nestmann, Frank; Sickendiek, Ursel (Hrsg.). Das Handbuch der Beratung. Band 1: Disziplinen und Zugänge. Tübingen. S. 257-267.

Kreps, Gary L. (1990). The Nature of Therapeutic Communication. In: Gumpert, Gary; Fish, Sandra L. (Eds.). Talking to Strangers. Mediated Therapeutic Communication. Norwood, NJ. S. 29-38.

Krotz, Friedrich (1996). Parasoziale Interaktion und Identität im elektronischen mediatisierten Kommunikationsraum. In: Vorderer, Peter (Hrsg.). Fernsehen als Beziehungskiste. Parasoziale Beziehungen und Interaktionen mit TV-Personen. Opladen. S. 73-90.

Krotz, Friedrich (2001). Die Mediatisierung kommunikativen Handelns. Wiesbaden.

Lampert, Claudia (2005). Gezielte Lebenshilfe durch Entertainment-Education? In: merz 49. Jg. Nr. 5. S. 33-37.

Lenz, Karl (2003). Zur sozialen Lage der Kinder im Freistaat Sachsen. Expertise für den Zweiten Sächsischen Kinder- und Jugendbericht 2003. In: Staatsministerium für Soziales, Freistaat Sachsen (Hrsg.). Expertisen zum Zweiten Sächsischen Kinder- und Jugendbericht. S. 7-47.

Marbach, Jan H. (2005). Soziale Netzwerke von Acht- bis Neunjährigen. Die Rolle von Sozialkapital in der Sozialisation von Kindern im Grundschulalter. In: Alt, Christian (Hrsg.). Kinderleben - Aufwachsen zwischen Familie, Freunden und Institutionen. Band 2: Aufwachsen zwischen Freunden und Institutionen. Wiesbaden. S. 83-121.

Mattusch, Uwe (1995). Fernsehprogramme für Teens. In: Erlinger, Dieter et al. (Hrsg.). Handbuch des Kinderfernsehens. Konstanz. S. 453-461.

Medienpädagogischer Forschungsverbund Südwest (Hrsg.) (2003). KIM-Studie 2003. Kinder und Medien. Computer und Internet. Basisuntersuchung zum Medienumgang 6- bis 13Jähriger in Deutschland. Baden-Baden.

Medienpädagogischer Forschungsverbund Südwest (Hrsg.) (2005). KIM-Studie 2005. Kinder und Medien. Computer und Internet. Basisuntersuchung zum Medienumgang 6- bis 13Jähriger in Deutschland. Baden-Baden.

Meves, Christa (1998). ABC der Lebensberatung. Grundlagen für die Begegnung mit Ratsuchenden. Neuhausen-Stuttgart.

Nestmann, Frank (2004). Beratungsmethoden und Beratungsbeziehung. In: Engel, Frank; Nestmann, Frank; Sickendiek, Ursel (Hrsg.). Das Handbuch der Beratung. Band 2: Ansätze, Methoden und Felder. Tübingen. S. 783-796.

Oerter, Rolf (1998). Kindheit. In: Oerter, Rolf, Montada, Leo (Hrsg.). Entwicklungspsychologie. 4., korrigierte Ausgabe. Weinheim. S. 249-309. 
Oerter, Rolf; Dreher, Eva (1998). Jugendalter. In: Oerter, Rolf; Montada, Leo: Entwicklungspsychologie. Weinheim. S. 311-395.

Poli, Daniel; Ertelt, Jürgen (2005). Beratung im Netz. Online-Pflaster oder Online-Therapie? In: merz, 49. Jg. Nr. 5. S. 44-47.

Paus-Haase, Ingrid (1998). Heldenbilder im Fernsehen. Untersuchung zur Symbolik von Serienfavoriten. Opladen, Wiesbaden.

Paus-Haase, Ingrid; Hasebrink, Uwe; Keuneke, Susanne; Krotz, Friedrich (1999). Talkshows im Alltag von Jugendlichen. Der tägliche Balanceakt zwischen Orientierung, Unterhaltung, Amüsement und Ablehnung. Olpladen.

Paus-Haase, Ingrid; Hasebrink, Uwe (2001). Talkshows im Alltag von Jugendlichen: Zusammenfassung der 〈Talkshow-Studie`. In: Göttlich, Udo; Krotz, Friedrich; Paus-Haase, Ingrid (Hrsg.). Daily Soaps und Daily Talks im Alltag von Jugendlichen. Opladen. S. 137-154.

Paus-Hasebrink, Ingrid (2004). Neue Kinder - neue Kindheiten? Zur Orientierung Heranwachsender in medialen Netzwerken. Perspektiven aus der Kindheits- und Jugendforschung. In: Grosse-Loheide, Mike; Hasebrink, Uwe (Hrsg.). Netzwerke für die Informationsgesellschaft. Bielefeld. S. 22-31.

Paus-Hasebrink, Ingrid; Bichler, Michelle (2005). Kindheit im Wandel - Bleiben sozial schwache Kinder auf der Strecke? Ein Plädoyer für die Intensivierung der Forschung zum Medienumgang von Kindern aus anregungsärmeren Milieus. In: televizion, 18/2005/2. S.104-107.

Salisch, Maria von (2005). Streit unter Freunden. Was tun Schulkinder, wenn sie sich ärgern? In: Alt, Christian (Hrsg.). Kinderleben - Aufwachsen zwischen Familie, Freunden und Institutionen. Band 2: Aufwachsen zwischen Freunden und Institutionen. Wiesbaden. S. 63-82.

Sander, Uwe (2002). Kinder und Medien. In: Deutsches Kinderhilfswerk e. V. (Hrsg.) Kinderreport 2002. München. S. 157-164.

Sanders, Rudolf (2004). Die Beziehung zwischen Ratsuchendem und Berater. In: Engel, Frank; Nestmann, Frank; Sickendiek, Ursel (Hrsg.). Das Handbuch der Beratung. Band 2: Ansätze, Methoden und Felder. Tübingen. S. 797-807.

Schneider, Regina (2002). Schulprobleme, Lebensprobleme, persönliche Krisen. Beratung als Orientierungshilfe. In: Pädagogik, 54. Jg. Nr.4. S. 34-37.

Schneider, Susanne (2005). Lernfreude und Schulangst. Wie es 8- bis 9-Jährigen Kindern in der Grundschule geht. In: Alt, Christian (Hrsg.). Kinderleben - Aufwachsen zwischen Familie, Freunden und Institutionen. Band 2: Aufwachsen zwischen Freunden und Institutionen. Wiesbaden, S. 199-230.

Schöppe, Arno (1998). Internet-Raststätte für Sex-Surfer? Digitale Beratung von Pro Familia. In: Janssen, Ludwig (Hrsg.). Auf der virtuellen Couch. Selbsthilfe, Beratung und Therapie im Internet. Bonn. S. 117-128.

Schorb, Bernd; Fleischer, Sandra; Lauber, Achim (2004). Wahrnehmung und Akzeptanz der Magazinsendung «Der KI.KA-Kummerkasten». Forschungsbericht im Auftrag des KI.KA. www.uni-leipzig.de/ schorb «http://www.uni-leipzig.de/ schorbs.

Schrödter, Wolfgang (2004). Ethische Richtlinien für Beratung. In: Engel, Frank; Nestmann, Frank; Sickendiek, Ursel (Hrsg.). Das Handbuch der Beratung. Band 1: Disziplinen und Zugänge. Tübingen. S. 453-467.

Schwarzer, Christine; Posse, Norbert (1993). Beratung. In: Weidenmann, Bernd u. a. (Hrsg.). Pädagogische Psychologie. 3. Auflage. München, Weinheim. S. 631-666. 
Schwarzer, Christine; Posse, Norbert (2004). Pädagogische Psychologie und Beratung. In: Engel, Frank; Nestmann, Frank; Sickendiek, Ursel (Hrsg.). Das Handbuch der Beratung. Band 1: Disziplinen und Zugänge. Tübingen. S. 73-87.

Süss, Daniel (2004). Mediensozialisation von Heranwachsenden. Dimensionen - Konstanten - Wandel. Wiesbaden.

Tetz, Margit (2000). «Da ist jemand, der mir zuhört...〉. Das Dr.-Sommer-Team: Zum Selbstverständnis der Aufklärung in BRAVO. In: Knoll, Joachim, H.; Monssen-Engberding, Elke (Hrsg.). Bravo, Sex und Zärtlichkeit. Mönchengladbach. S. 96-109.

Theunert, Helga (1996a). ¿Da kann ich lernen, was ich nicht machen soll〉. Kinder rezipieren Reality-TV. In: Schorb, Bernd; Stiehler, Hans-Jörg (Hrsg.). Medienlust- Medienlast. Was bringt die Rezipientenforschung dem Rezipienten? München. S. 17-30.

Theunert, Helga (2005). Medien als Orte informellen Lernens im Prozess des Heranwachsens. In: Sachverständigenkommission Zwölfter Kinder- und Jugendbericht (Hrsg.). Kompetenzerwerb von Kindern und Jugendlichen im Schulalter. Band 3. München. S. 175-300.

Theunert, Helga; Gebel, Christa (Hrsg.) (2000). Lehrstücke fürs Leben in Fortsetzung. Serienrezeption zwischen Kindheit und Jugend. München.

Traub, Angelika (2005). Ein Freund, ein guter Freund... Die Gleichaltrigenbeziehungen der 8- bis 9-Jährigen. In: Alt, Christian (Hrsg.). Kinderleben - Aufwachsen zwischen Familie, Freunden und Institutionen. Band 2: Aufwachsen zwischen Freunden und Institutionen. Wiesbaden. S. 23-62.

Vogel, Heiner (2004). Qualitätssicherung von Beratung. In: Engel, Frank; Nestmann, Frank; Sickendiek, Ursel (Hrsg.). Das Handbuch der Beratung. Band 2: Ansätze, Methoden und Felder. Tübingen. S. 837-856.

Vorderer, Peter (1996). Picard, Brinkmann, Derrick \& Co. als Freunde der Zuschauer. Eine explorative Studie über parasoziale Beziehungen zu Serienfiguren. In: Vorderer, Peter (Hrsg.). Fernsehen als Beziehungskiste. Parasoziale Beziehungen und Interaktionen mit TV-Personen. Opladen. S. 153-171.

Vorheyer, Claudia (2005). Wer gehört zur Familie? Strukturelle Eigenschaften der familialen Netzwerke von Kindern. In: Alt, Christian (Hrsg.). Kinderleben - Aufwachsen zwischen Familie, Freunden und Institutionen. Band 1: Aufwachsen in Familien. Wiesbaden. S. 23-44.

Vossler, Andreas (2005). Das Jahrhundert der Beratung. Entwicklung und gesellschaftliche Bedeutung von Beratungsangeboten. In: merz, 49. Jg. Nr. 5. S. 9-14.

Wahler, Eva-Maria (2004). BRAVO TV im ZDF. Starmagazin und Jugendserie. In: televizion, 17/2. S. 53-55.

Zackl, Daniela (2005). Dr.MAG love Oder: «Liebe ist keine Nebensache〉. In: televizion, 18/1. S. 7-8. 